\title{
On an Additive Schwarz Preconditioner for the Crouzeix-Raviart Mortar Finite Element
}

Talal Rahman ${ }^{1}$, Xuejun $\mathrm{Xu}^{2}$, and Ronald H.W. Hoppe ${ }^{3}$

1 University of Augsburg, Department of Mathematics, Universitätsstr. 14, D-86159 Augsburg, Germany (talal.rahman@math.uni-augsburg.de)

2 LSEC, Institute of Computational Mathematics, Chinese Academy of Sciences, P.O.Box 2719, Beijing, 100080, People's Republic of China.

(xxj@lsec.cc.ac.cn)

3 Department of Mathematics, University of Houston, Calhoun Street, Houston, TX-77204-3008, U.S.A., (rohop@math.uh.edu) and Institute for Mathematics, University of Augsburg, Universitätsstr. 14, D-86159 Augsburg, Germany.

(hoppe@math.uni-augsburg.de)

Summary. We consider an additive Schwarz preconditioner for the algebraic system resulting from the discretization of second order elliptic equations with discontinuous coefficients, using the lowest order Crouzeix-Raviart element on nonmatching meshes. The overall discretization is based on the mortar technique for coupling nonmatching meshes. A convergence analysis of the preconditioner has recently been given in Rahman et al. [2003]. In this paper, we give a matrix formulation of the preconditioner, and discuss some of its numerical properties.

\section{Introduction}

We consider the Crouzeix-Raviart (CR), or the nonconforming P1 finite element discretization on nonmatching meshes of the following elliptic problem with discontinuous coefficients: Find $u^{*} \in H_{0}^{1}(\Omega)$ such that

$$
a\left(u^{*}, v\right)=f(v), v \in H_{0}^{1}(\Omega),
$$

where $\Omega \subset R^{2}$ is a bounded, simply connected polygonal domain, $a(u, v)=$ $\sum_{i=1}^{N} \rho_{i}(\nabla u, \nabla v)_{L^{2}\left(\Omega_{i}\right)}$ and $f(v)=\sum_{i=1}^{N} \int_{\Omega_{i}} f v d x$, and $\bar{\Omega}=\cup_{i=1}^{N} \bar{\Omega}_{i}$ is the partition of $\Omega$ into nonoverlapping polygonal subdomains $\Omega_{i}$ of diameter $H_{i}$. The coefficients $\rho_{i}$ are positive constants with possibly large jumps across subdomain interfaces. Let $X_{h}\left(\Omega_{i}\right)$ be the nonconforming P1 (Crouzeix-Raviart) finite element space defined on a quasi-uniform triangulation $\mathcal{T}_{h}\left(\Omega_{i}\right)$ of mesh size $h_{i}$, of the subdomain $\Omega_{i}$, consisting of functions which are piecewise linear in each triangle $\tau \subset \Omega_{i}$, continuous at the interior edge midpoints $x_{k} \in \Omega_{i h}^{C R}$, and vanishing at the edge midpoints lying on the boundary $\partial \Omega$. 
Since the triangulations on $\Omega_{i}$ and $\Omega_{j}$ may not match on their common interface $\bar{\Gamma}_{i j}=\bar{\Omega}_{i} \cap \bar{\Omega}_{j}$, the functions in $X_{h}(\Omega)=\Pi_{i=1}^{N} X_{h}\left(\Omega_{i}\right)$ are discontinuous at edge midpoints along the interface. We use a special technique, known as the mortar technique, cf. Bernardi et al. [1994], for the coupling of nonmatching meshes. An analysis of the mortar technique for the Crouzeix-Raviart element has been given in Marcinkowski [1999].

According to the mortar technique, a weak continuity condition, called the mortar condition, is imposed on the function along the interfaces. A function $u_{h}=\left\{u_{i}\right\}_{i=1}^{N} \in X_{h}$ satisfies the mortar condition on the interface $\Gamma_{i j}$, if $Q_{m} u_{i}=Q_{m} u_{j}$, where $Q_{m}: L^{2}\left(\Gamma_{i j}\right) \rightarrow M^{h_{j}}\left(\delta_{m(j)}\right)$ is the $L^{2}$-projection operator defined as: $\left(Q_{m} u, \psi\right)_{L^{2}\left(\delta_{m(j)}\right)}=(u, \psi)_{L^{2}\left(\delta_{m(j)}\right)}, \quad \forall \psi \in M^{h_{j}}\left(\delta_{m(j)}\right)$, where $\delta_{m(j)} \subset \partial \Omega_{j}$ is the nonmortar side of $\Gamma_{i j}, M^{h_{j}}\left(\delta_{m(j)}\right) \subset L^{2}\left(\Gamma_{i j}\right)$ is the test space of functions which are piecewise constant on the triangulation of $\delta_{m(j)}$, and $(\cdot, \cdot)_{L^{2}\left(\delta_{m(j)}\right)}$ denotes the $L^{2}$ innerproduct on $L^{2}\left(\delta_{m(j)}\right)$. The other side of $\Gamma_{i j}$, called the mortar side, is denoted by $\gamma_{m(i)} \subset \partial \Omega_{i}$. The discrete problem takes the following form: Find $u_{h}^{*}=\left\{u_{i}\right\}_{i=1}^{N} \in V_{h}$ such that

$$
a_{h}\left(u_{h}^{*}, v_{h}\right)=f\left(v_{h}\right), \quad \forall v_{h} \in V_{h},
$$

where $V_{h} \subset X_{h}$ is a subspace of functions which satisfy the mortar condition on all interfaces, and $a_{h}(u, v)=\sum_{i=1}^{N} \rho_{i} \sum_{\tau \in \mathcal{T}_{h}\left(\Omega_{i}\right)}(\nabla u, \nabla v)_{L^{2}(\tau)}=$ $\sum_{i=1}^{N} a_{i}(u, v) . V_{h}$ is a Hilbert space with an inner product defined by $a_{h}(\cdot, \cdot)$. The problem has a unique solution and a priori error estimates have been provided in Marcinkowski [1999].

Even though, there exists a lot of work concerning the nonconforming P1 element on matching grids, cf., e.g., Brenner [1996], Hoppe and Wohlmuth [1995], Sarkis [1997], the work on nonmatching grids is very limited, cf., e.g., Marcinkowski [1999], Xu and Chen [2001]. Recently, an efficient additive Schwarz method for the nonconforming P1 element on nonmatching grids has been proposed in Rahman et al. [2003]. In this paper, we complement the work by introducing the matrix formulation of the preconditioner, and discuss some of its numerical properties.

\section{An additive Schwarz preconditioner}

In this section, we describe the additive Schwarz preconditioner of Rahman et al. [2003], for the problem (2), which is based on the idea of solving local subproblems on nonoverlapping subdomains, and coarse problems on specially constructed subspaces of small dimensions. The preconditioner is defined using the general framework for additive Schwarz methods, cf. Smith et al. [1996]. We decompose $V_{h}$ as $V_{h}=\sum_{\gamma} V^{\gamma}+V^{0}+\sum_{i=1}^{N} V^{i}$, where the first sum is taken over the set of all mortar sides $\{\gamma\}$. For $i=1, \cdots, N, V^{i}$ is the restriction of $V_{h}$ to $\Omega_{i}$, with functions vanishing at subdomain boundary edge midpoints $\partial \Omega_{i h}^{C R}$ as well as on the remaining subdomains. $V^{\gamma}$ is a space of 

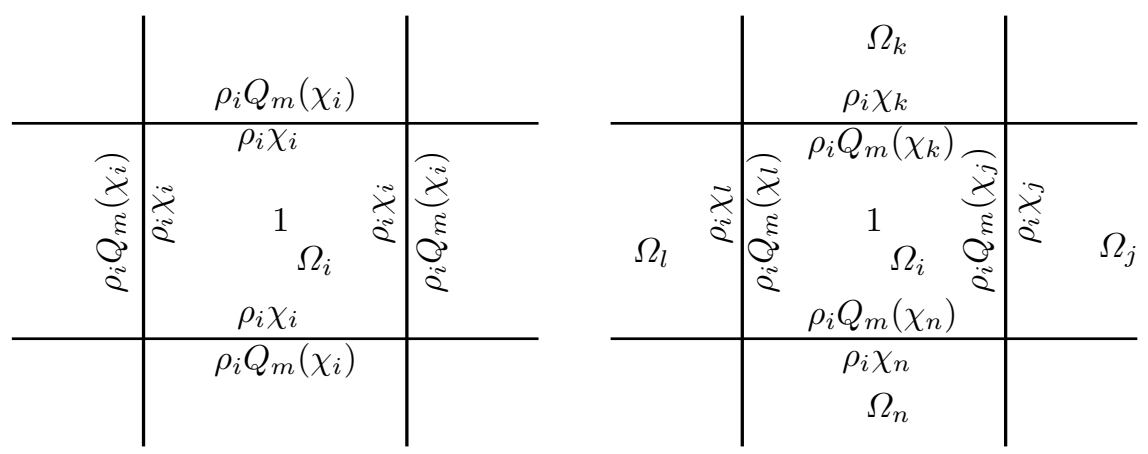

Fig. 1. Examples of $\Phi_{i}$ corresponding to a subdomain $\Omega_{i}$ having only mortar sides (left) or nonmortar sides (right), indicating the nonzero values of the function.

functions given by their values on mortar edge midpoints $\gamma_{h}^{C R}, V^{\gamma}=\{v \in$ $\left.V_{h}: v(x)=0, x \in \bar{\Omega}_{h}^{C R} \backslash \gamma_{h}^{C R}\right\}$. The coarse space $V^{0}$, a special space having a dimension equal to the number of subdomains, is defined using the function $\chi_{i} \in X_{h}\left(\Omega_{i}\right)$ associated with the subdomain $\Omega_{i} . \chi_{i}$ is defined by its nodal values as: $\chi_{i}(x)=1 / \sum_{j} \rho_{j}(x)$ at $x \in \bar{\Omega}_{i h}^{C R}$, where the sum is taken over the subdomains which $x$ is common to, $V^{0}$ is given as the span of its basis functions, $\Phi_{i}, i=1, \cdots, N$, i.e., $V^{0}=\operatorname{span}\left\{\Phi_{i}: i=1, \cdots, N\right\}$, where $\Phi_{i}$ associated with $\Omega_{i}$, is defined as follows (cf. Figure 1 ).

$$
\Phi_{i}(x)=\left\{\begin{array}{cl}
1, & x \in \Omega_{i h}^{C R}, \\
\rho_{i} \chi_{i}(x), & x \in \gamma_{m(i) h}^{C R}, \\
\rho_{i} Q_{m}\left(\chi_{j}\right)(x), & x \in \delta_{m(i) h}^{C R}, \delta_{m(i)}=\gamma_{m(j)}, \\
\rho_{i} Q_{m}\left(\chi_{i}\right)(x), & x \in \delta_{m(j) h}^{C R}, \delta_{m(j)}=\gamma_{m(i)}, \\
\rho_{i} \chi_{j}(x), & x \in \gamma_{m(j) h}^{C R}, \gamma_{m(j)}=\delta_{m(i)}, \\
0, & x \in \partial \Omega_{i h}^{C R} \cap \partial \Omega,
\end{array}\right.
$$

and $\Phi_{i}(x)=0$ at all other $x$ in $\bar{\Omega}_{h}^{C R}$. We use exact bilinear forms for all our subproblems. The projection like operators $T^{i}: V_{h} \rightarrow V^{i}$ are defined in the standard way, i.e., for $i \in\{\{\gamma\}, 0, \cdots, N\}$ and $u \in V_{h}, T^{i} u \in V^{i}$ is the solution of $a_{h}\left(T^{i} u, v\right)=a_{h}(u, v), v \in V^{i}$. Let $T=\sum_{\gamma} T^{\gamma}+T^{0}+T^{1}+\cdots+T^{N}$. The problem (2) is now replaced by the following preconditioned system,

$$
T u_{h}^{*}=g
$$

where $g=\sum_{\gamma} T^{\gamma} u_{h}^{*}+\sum_{i=0}^{N} T^{i} u_{h}^{*}$. Let $c$ and $C$ represent generic constants independent of the mesh sizes $h=\inf _{i} h_{i}$ and $H=\max _{i} H_{i}$, and of the jumps of the coefficients $\rho_{i}$, then the following result holds.

Theorem 1 (Rahman et al. [2003]). For all $u \in V_{h}$,

$$
c \frac{h}{H} a_{h}(u, u) \leq a_{h}(T u, u) \leq C a_{h}(u, u) .
$$


The proof of this theorem is given in Rahman et al. [2003], which uses the general theory for Schwarz methods, cf. Smith et al. [1996]. It follows from the theorem, that the condition number of the operator $T$ is bounded by $c\left(\frac{H}{h}\right)$.

\subsection{Matrix formulation}

Our aim is to derive a matrix representation for the preconditioned system (4). The finite element space $V^{h}$ can be expressed as $V^{h}=\operatorname{span}\left\{\phi_{k}\right\}$, where each basis function $\phi_{k}$ is associated with a node $x_{k}$ which is either a subdomain interior edge midpoint or a mortar edge midpoint. Let $\varphi_{k}^{(i)}$ denote the standard nodal basis function of $X_{h}\left(\Omega_{i}\right)$, associated with the edge midpoint $x_{k}$. The basis functions are defined as follows.

If $x_{k} \in \Omega_{i h}^{C R}$, a subdomain interior node, then $\phi_{k}(x)$ is exactly equal to $\varphi_{k}(x)$. If $x_{k} \in \gamma_{m(i) h}^{C R}$, a mortar node, then $\phi_{k}(x)=\varphi_{k}(x)$ on $\bar{\Omega}_{i}$, while on $\bar{\delta}_{m(j)}$, where $\gamma_{m(i)}=\delta_{m(j)}, \phi_{k}(x)=Q_{m}\left(\varphi_{k}\right)(x)$ at $x \in \delta_{m(j) h}^{C R}$. $\phi_{k}$ is zero at the remaining edge midpoints of $\bar{\Omega}_{j}$, and zero everywhere on the remaining subdomains. Note that there are no basis functions associated with nonmortar edge midpoints. Using these basis functions of $V_{h}$, the problem (2) can be rewritten in the matrix form as

$$
\mathrm{Au}^{*}=\mathbf{f},
$$

where $\mathbf{u}^{*}$ is a vector of nodal values of $u_{h}^{*}$, and $\mathbf{A}$ is a matrix generated by the bilinear form $a_{h}(.,$.$) on V_{h} \times V_{h}$. We shall now see how this matrix can be obtained from the local matrices $\hat{\mathbf{E}}_{i}$ generated by $a_{i}(.,$.$) on X_{h}\left(\Omega_{i}\right) \times X_{h}\left(\Omega_{i}\right)$.

Observing that $a_{h}(.,)=.\sum_{i=1}^{N} a_{i}(.,$.$) , where a_{i}(.,)=.\left.a_{h}(.,)\right|_{.\Omega_{i}}$, we can calculate the elements of $\mathbf{A}$ from their local contributions restricted to individual subdomains $\Omega_{i}$. In order to calculate the local contribution $a_{i}(.,$.$) ,$ we use only those basis functions that have nonzero supports on $\bar{\Omega}_{i}$. These basis functions are exactly the ones associated with the nodes of $\Omega_{i h}^{C R}, \gamma_{m(i) h}^{C R}$ $\left(\gamma_{m(i)} \subset \partial \Omega_{i}\right)$, and the set $\gamma_{m(j) h}^{C R}\left(\gamma_{m(j)}=\delta_{m(i)} \subset \partial \Omega_{i}\right)$ of neighboring mortar edge midpoints except those on $\partial \Omega$. Let $\Lambda_{i}$ be the set of all these nodes.

Let $\mathbf{P}_{i}$ be the restriction matrix which is a permutation of a rectangular identity matrix, such that $\mathbf{P}_{i} \mathbf{u}$ returns the vector of all coefficients of $\mathbf{u}$, associated with the nodes of $\Lambda_{i} . \mathbf{P}_{i}^{T}$ is the corresponding extension matrix. Let $\mathbf{E}_{i}$, associated with the subdomain $\Omega_{i}$, be the matrix generated by $a_{i}(.,$.$) on$ $\operatorname{span}\left\{\phi_{k}: x_{k} \in \Lambda_{i}\right\} \times \operatorname{span}\left\{\phi_{l}: x_{l} \in \Lambda_{i}\right\}$. Using these three types of matrices, we can assemble the global matrix as $\mathbf{A}=\sum_{i=1}^{N} \mathbf{P}_{i}^{T} \mathbf{E}_{i} \mathbf{P}_{i}$.

We note that $\mathbf{E}_{i}=\left\{a_{i}\left(\phi_{k}, \phi_{l}\right)\right\}$, where $x_{k}, x_{l} \in \Lambda_{i}$, and $\hat{\mathbf{E}}_{i}=\left\{a_{i}\left(\varphi_{k}, \varphi_{l}\right)\right\}$, where $x_{k}, x_{l} \in \bar{\Omega}_{i h}^{C R}$. If $x_{k}, x_{l} \in \Omega_{i h}^{C R} \cup \gamma_{m(i) h}^{C R}$, then $a_{i}\left(\phi_{k}, \phi_{l}\right)=a_{i}\left(\varphi_{k}, \varphi_{l}\right)$. If $x_{k} \in \gamma_{m(j) h}^{C R}$, then the calculation of an element of $\mathbf{E}_{i}$ involving $\phi_{k}$, requires the values of $Q_{m}\left(\varphi_{k}\right)\left(x_{o}\right)$ at the nodes $x_{o} \in \delta_{m(i) h}^{C R}$, since, by definition, $\phi_{k}=$ $\sum_{x_{o} \in \delta_{m(i) h}^{C R}} Q_{m}\left(\varphi_{k}\right)\left(x_{o}\right) \varphi_{o}$ in $\bar{\Omega}_{i}$. In the following, we derive these coefficients $\left\{Q_{m}\left(\varphi_{k}\right)\left(x_{o}\right)\right\}$ from the mortar condition. 
We assume that the subdomain $\Omega_{i}$ has only one nonmortar side $\delta_{m(i)}$, the extension to more than one nonmortar edge is straightforward. Let $\mathbf{M}_{\gamma_{m(j)}}$ $=\left\{\left(\varphi_{k}, \psi_{o}\right)_{L^{2}\left(\delta_{m(i)}\right)}\right\}$ and $\mathbf{S}_{\delta_{m(i)}}=\left\{\left(\varphi_{o}, \psi_{o}\right)_{L^{2}\left(\delta_{m(i)}\right)}\right\}$, for $x_{k} \in \gamma_{m(j) h}^{C R}$ and $x_{o} \in \delta_{m(i) h}^{C R}$, be the master and the slave matrix, respectively. Then

$$
\mathbf{Q}_{m(i)}=\mathbf{S}_{\delta_{m(i)}}^{-1} \mathbf{M}_{\gamma_{m(j)}}
$$

is the matrix representation of the mortar projection $Q_{m}$. The columns of this matrix correspond to the nodes $x_{k} \in \gamma_{m(j) h}^{C R}$, containing exactly the coefficients $\left\{Q_{m}\left(\varphi_{k}\right)\left(x_{o}\right)\right\}$. We note that $\mathbf{S}_{\delta_{m(i)}}$ is a diagonal matrix containing the lengths of the edges along $\delta_{m(i)}$, as entries.

Now define the matrix $\mathbf{Q}_{i}=\operatorname{diag}\left(\mathbf{I}, \mathbf{Q}_{m(i)}\right)$, where $\mathbf{I}$ is the identity matrix corresponding to the nodes of $\Omega_{i h}^{C R}$ and $\gamma_{m(i) h}^{C R}$, and $\mathbf{Q}_{m(i)}$ is the projection matrix corresponding to the nodes of $\gamma_{m(j) h}^{C R}$. Then it is easy to see that $\mathbf{E}_{i}=$ $\mathbf{Q}_{i}^{T} \hat{\mathbf{E}}_{i} \mathbf{Q}_{i}$. Finally, we have $\mathbf{A}=\sum_{i=1}^{N} \mathbf{P}_{i}^{T} \mathbf{Q}_{i}^{T} \hat{\mathbf{E}}_{i} \mathbf{Q}_{i} \mathbf{P}_{i}$. In the same way, we get $\mathbf{f}=\sum_{i=1}^{N} \mathbf{P}_{i}^{T} \mathbf{Q}_{i}^{T} \hat{\mathbf{f}}_{i}$.

We follow the standard procedure, cf. Smith et al. [1996], for expressing the preconditioned system in matrix form. Since $V^{i} \subset V_{h}$, for $i \in$ $\{\{\gamma\}, 0,1, \cdots, N\}$, the interpolation operators $I_{i}: V^{i} \rightarrow V_{h}$ are simply the imbedding operators. Let $\mathbf{R}_{i}^{T}$ be the matrix representation of $I_{i}$. In matrix form, $T^{i}$ is then given by $\mathbf{T}_{i}=\mathbf{R}_{i}^{T} \mathbf{A}_{i}^{-1} \mathbf{R}_{i} \mathbf{A}$, where $\mathbf{A}_{i}=\mathbf{R}_{i} \mathbf{A} \mathbf{R}_{i}^{T}$. Now, setting $\mathbf{T}=\sum_{\gamma} \mathbf{T}_{\boldsymbol{\gamma}}+\sum_{i=0}^{N} \mathbf{T}_{i}$, which is the matrix representation of $T$, the preconditioned system (4) takes the following matrix form.

$$
\mathbf{T u}^{*}=\mathrm{g} .
$$

In this, $\mathbf{T}=\mathbf{B A}$ and $\mathbf{g}=\mathbf{B f}$, where $\mathbf{B}=\sum_{\gamma} \mathbf{R}_{\gamma}^{T} \mathbf{A}_{\gamma}^{-1} \mathbf{R}_{\gamma}+\sum_{i=0}^{N} \mathbf{R}_{i}^{T} \mathbf{A}_{i}^{-1} \mathbf{R}_{i}$ is the preconditioner. The restriction matrices $\mathbf{R}_{i}, i=1, \cdots, N$, and $\mathbf{R}_{\gamma}$ are all permutations of rectangular identity matrices such that $\mathbf{R}_{i} \mathbf{u}$ and $\mathbf{R}_{\gamma} \mathbf{u}$ return vectors of coefficients of $\mathbf{u}$, associated with the subdomain interior edge midpoints $\Omega_{i h}^{C R}$ and the mortar edge midpoints $\gamma_{h}^{C R}$, respectively. The construction of $\mathbf{R}_{0}$ is different but simple. Let $\mathbf{v}_{i}$ be the vector of nodal values of $\Phi_{i}$, then the columns of $\mathbf{R}_{0}^{T}$ consist of exactly these vectors, i.e., $\mathbf{v}_{i}, i=$ $1, \cdots, N$. The matrices $\mathbf{P}_{i}$ and $\mathbf{R}_{i}, i=1, \cdots, N$, and $\mathbf{R}_{\gamma}$ are never formed in practice. Their use in this section has been merely for the representation.

Most iterative methods for solving (7) require the actions of $\mathbf{A}$ and $\mathbf{B}$ on different vectors in each iteration. Once the matrices $\mathbf{Q}_{m(i)}$ and $\hat{\mathbf{E}}_{i}$, $i=1, \cdots, N$, and $\mathbf{A}_{i}, i \in\{\{\gamma\}, 0, \cdots, N\}$, are generated, the actions of $\mathbf{A}$ and $\mathbf{B}$ on the vectors can be calculated by multiplying the vectors with the expressions of $\mathbf{A}$ and $\mathbf{B}$, respectively.

\section{Numerical Examples}

In this section, we present numerical results and discuss some of the properties of the preconditioner presented in the previous section. The Preconditioned 
Table 1. Numerical results for varying jumps in the coefficients. $d \times d=36$ subdomains, each having $2 m^{2}=72$ or $2 n^{2}=50$ elements, are used for the triangulation.

\begin{tabular}{ccccc}
\hline $\begin{array}{c}\text { Coefficient } \\
\text { jump } \rho\end{array}$ & $\begin{array}{c}\text { Condition } \\
\text { number } \kappa_{2}\end{array}$ & $\begin{array}{c}\text { CG-iteration } \\
\text { counts }\end{array}$ & $\begin{array}{c}L^{2} \text {-norm of } \\
\text { error }\end{array}$ & $\begin{array}{c}H_{h}^{1} \text {-seminorm of } \\
\text { error }\end{array}$ \\
\hline $10^{0}$ & 31.580 & 35 & $0.9516 \cdot 10^{-3}$ & $0.4366 \cdot 10^{-1}$ \\
$10^{2}$ & 32.755 & 39 & $0.1099 \cdot 10^{-2}$ & $0.4558 \cdot 10^{-1}$ \\
$10^{4}$ & 32.825 & 39 & $0.1104 \cdot 10^{-2}$ & $0.4565 \cdot 10^{-1}$ \\
$10^{6}$ & 32.834 & 39 & $0.1104 \cdot 10^{-2}$ & $0.4565 \cdot 10^{-1}$ \\
\hline
\end{tabular}

Conjugate Gradients (PCG) method has been used for solving the preconditioned system (4), which stops as the relative norm of the residual drops below the tolerance $10^{-6}$. In all our experiments, a uniform triangulation has been used in each subdomain employing, in a checker board order, either $2 m^{2}$ or $2 n^{2}$ triangular elements, where $m$ and $n$ are chosen differently in order to have nonmatching grids with different mesh sizes across subdomain interfaces.

\section{Test I}

The objective of this test is to study the effect of coefficient jumps on the convergence. We consider a model problem for which the exact solution is known. The problem is defined on a unit square initially defined as the union of $2 \times 2$ nonoverlapping square subregions with coefficients $\rho_{1}=\rho, \rho_{2}=1, \rho_{3}=$ 1 , and $\rho_{4}=\rho$ so that the jump in the coefficients across any subregion interface is equal to $\rho>0$. The function $f$ is chosen such that the exact solution can be given by $u(x, y)=\sin (\pi x) \sin (\pi y)$. Note that $\nabla u \cdot \eta$ vanishes along the subregion interfaces.

Numerical results for varying jumps in the coefficients are presented in Table 1 , showing condition number estimate of the preconditioned system, and the $L^{2}$-norm and the broken $H^{1}$-seminorm $\left(H_{h}^{1}\right)$ of the error in the numerical solution. The condition number estimates, as shown in the table, remain unchanged as the jump increases, illustrating that the preconditioner is robust with respect to jumps in the coefficients. In Table 2 , we present the weighted $L^{2}$-norm $\left(L_{\rho}^{2}\right)$ and the weighted broken $H^{1}$-seminorm $\left(H_{\rho h}^{1}\right)$ of the error for varying subdomain size and mesh size, where the weights are the coefficients

Table 2. Numerical results for varying subdomain size and mesh size, and fixed $\rho=10 . H$ and $h$ of the second row correspond to $d=4, m=12$ and $n=11$.

\begin{tabular}{cccccc}
\hline $\begin{array}{c}\text { Subdomain } \\
\text { size }\end{array}$ & size & number $\kappa_{2}$ & $\begin{array}{c}\text { Condition } \\
\text { numts }\end{array}$ & $\begin{array}{c}L_{\rho}^{2} \text {-norm of } \\
\text { error }\end{array}$ & $\begin{array}{c}H_{\rho h}^{1} \text {-seminorm of } \\
\text { error }\end{array}$ \\
\hline$H$ & $\frac{1}{2} h$ & 131.27 & 68 & $0.2541 \cdot 10^{-3}$ & $0.2914 \cdot 10^{-1}$ \\
$H$ & $h$ & 65.08 & 47 & $0.1056 \cdot 10^{-2}$ & $0.6057 \cdot 10^{-1}$ \\
$\frac{1}{2} H$ & $\frac{1}{2} h$ & 66.20 & 53 & $0.3778 \cdot 10^{-3}$ & $0.3293 \cdot 10^{-1}$ \\
\hline
\end{tabular}


$\rho_{i}$. The condition number estimates of the table are in accordance with the theory. The error in the $L_{\rho}^{2}$-norm and $H_{\rho h^{-}}^{1}$-seminorm indicate convergence as $O\left(h^{2}\right)$ and $O(h)$, respectively.

\section{Test II}

In our second test, we consider the choice of mortar or nonmortar sides, and the ratio between mesh sizes from neighboring subdomains, and discuss their possible influence on the convergence. The problem is defined on a unit square, with the force function $f(x)=2 \pi^{2} \sin (\pi x) \sin (\pi x)$. We assume the domain to be the union of $d \times d$ subregions (subdomains) with coefficients $\rho_{i}=1$ or $\rho_{i}=\rho=10^{4}$ distributed in a checkerboard order.

Table 3. Condition number and CG-iteration counts (in parentheses) for two opposite choices of mortar sides and varying mesh size ratio. $m=12$ for $\rho_{i}=\rho$, and $n=11,6$ for $\rho_{i}=1$ giving $h_{\rho_{i}=\rho} / h_{\rho_{i}=1} \approx 1, \frac{1}{2}$.

\begin{tabular}{ccccc}
\hline Subdomains & \multicolumn{2}{c}{ Choice I } & \multicolumn{2}{c}{ Choice II } \\
$d \times d$ & $m=12, n=11$ & $m=12, n=6$ & $m=12, n=11$ & $m=12, n=6$ \\
\hline $6 \times 6$ & $68.63(57)$ & $68.63(54)$ & $68.62(57)$ & $63.28(49)$ \\
$9 \times 9$ & $68.79(59)$ & $68.95(55)$ & $68.72(57)$ & $63.40(49)$ \\
\hline
\end{tabular}

Each column in Table 3 and Table 4, corresponds to a fixed pair $\{m, n\}$ representing a fixed $\frac{H}{h}$ ratio. Two opposite choices of mortar sides, called 'Choice I' and 'Choice II', have been chosen for the experiment. 'Choice I' corresponds to choosing the sides with larger coefficients as the mortar sides, and 'Choice II' corresponds to the opposite choice. Under each choice of mortar sides, two sets of results corresponding to different mesh size ratios between neighboring subdomains are presented. The difference between the tables is as follows: In Table 3, we use a finer mesh on subdomains with larger coefficient, i.e. $h_{\rho_{i}=\rho}<h_{\rho_{i}=1}$, (this gives a better a priori error, cf. Bernardi and Verfürth [2000]), and in Table 4, we do the opposite.

Table 4. Condition number estimate and iteration counts (in parentheses) for two opposite choices of mortar sides and varying mesh size ratio. $m=11,6$ for $\rho_{i}=\rho$, and $n=12$ for $\rho_{i}=1$ giving $h_{\rho_{i}=1} / h_{\rho_{i}=\rho} \approx 1, \frac{1}{2}$.

\begin{tabular}{ccccc}
\hline Subdomains & \multicolumn{3}{c}{ Choice II } & \multicolumn{2}{c}{ Choice I } \\
$d \times d$ & $m=11, n=12$ & $m=6, n=12$ & $m=11, n=12$ & $m=6, n=12$ \\
\hline $6 \times 6$ & $62.86(55)$ & $33.78(45)$ & $62.87(57)$ & $33.77(43)$ \\
$9 \times 9$ & $62.99(51)$ & $34.04(42)$ & $62.99(51)$ & $34.05(40)$ \\
\hline
\end{tabular}


As seen from the tables above, for a particular choice of mortar sides and a fixed $\frac{H}{h}$ ratio, the condition number estimates remain bounded. In fact, the choice of mortar sides show no or only a mild influence on the condition number estimates. The place where this mild influence is seen in the table, is for the mesh size ratio $h_{\rho_{i}=\rho} / h_{\rho_{i}=1}=\frac{1}{2}$, cf. Table 3 . It has however been observed through experiments that the percentile difference between the estimates reduces gradually with the mesh size. We close this section by making a final remark on Table 4 . As seen from the table, the condition number estimates for the ratio $h_{\rho_{i}=1} / h_{\rho_{i}=\rho}=\frac{1}{2}$ are approximately half of those for $h_{\rho_{i}=1} / h_{\rho_{i}=\rho}=1$. This is due to the minimum eigenvalue. It is not difficult to show, taking into account the special shapes of the basis functions $\left\{\Phi_{i}\right\}$ (due to the checkerboard distribution of the coefficients) in the proof of Theorem 1 , that the bound for the minimum eigenvalue approximately doubles as the ratio is halved.

\section{References}

C. Bernardi, Y. Maday, and A. T. Patera. A new non conforming approach to domain decomposition: The mortar element method. In H. Brezis and J.-L. Lions, editors, Collège de France Seminar. Pitman, 1994. This paper appeared as a technical report about five years earlier.

C. Bernardi and R. Verfürth. Adaptive finite element methods for elliptic equations with nonsmooth coefficients. Numerische Mathematik, 85(4):579$608,2000$.

S. C. Brenner. Two-level additive Schwarz preconditioners for nonconforming finite element methods. Mathematics of Computation, 65(215):897-921, 1996.

R. H. W. Hoppe and B. Wohlmuth. Adaptive multilevel iterative techniques for nonconforming finite element discretizations. East-West J. Numer. Math., 3:179-197, 1995.

L. Marcinkowski. The mortar element method with locally nonconforming elements. BIT, 39:716-739, 1999.

T. Rahman, X. Xu, and R. Hoppe. An additive Schwarz method for the Crouzeix-Raviart finite element for elliptic problems discontinuous coefficients. Submitted to Numerische Mathematik, 2003.

M. Sarkis. Nonstandard coarse spaces and Schwarz methods for elliptic problems with discontinuous coefficients using non-conforming element. $\mathrm{Nu}$ merische Mathematik, 77:383-406, 1997.

B. F. Smith, P. E. Bjørstad, and W. Gropp. Domain Decomposition: Parallel Multilevel Methods for Elliptic Partial Differential Equations. Cambridge University Press, 1996.

$\mathrm{X}$. $\mathrm{Xu}$ and J. Chen. Multigrid for the mortar element method for the P1 nonconforming element. Numerische Mathematik, 88(2):381-398, 2001. 